\title{
SIMPLE DESIGN OF MULTIPLE AERODYNAMIC PLASMA ACTUATOR
}

\author{
S. L. Chernyshev, M. D. Gamirullin, A. P. Kuryachii, \\ and V.M. Litvinov
}

Central Aerohydrodynamic Institute (TsAGI)

1 Zhukovsky Str., Zhukovsky, Moscow Region 140180, Russia

\begin{abstract}
A new simple design of multiple dielectric barrier discharge (DBD) actuator intended for gas flow acceleration in thin boundary layer on lengthy aerodynamic surface is proposed. The operability of the considered multi-DBD-actuator is confirmed experimentally. Proposed method of measurements of the electric power consumed by the actuator permits to estimate this value simply and with acceptable accuracy. The thrust generated by DBD-actuator and thrust-to-power relation were estimated owing to pressure probe measurements of the induced flow velocity.
\end{abstract}

\section{INTRODUCTION}

At present significant attention is given to study of possible applications of plasma actuators for gas flow control on aerodynamic surfaces [1-4]. For example, laminar-to-turbulent transition delay in boundary layer on airplane wing is one of the effective methods of friction drag reduction and, consequently, fuel consumption and atmospheric pollution decrease. Electrogasdynamic method of laminar flow control (EGD-LFC) on a swept wing proposed at TsAGI [5, 6] implies volumetric force impact and, as a consequence, boundary layer flow acceleration in small vicinity of a wing leading edge along the whole of the edge. Dielectric barrier discharge actuators in series [7] seem to be suitable for creation necessary force impact over a lengthy part of a wing surface. But it must be taken into account that the boundary layer thickness near the swept wing leading edge of typical civil airplane at cruise flight conditions is very small (less than $0.5 \mathrm{~mm}$ ) [8]. This circumstance demands the use of diminutive enough actuators. At the same time, the cross-talk phenomenon between the adjacent electrodes of actuators in series reduces the advantage of the multiple DBDs concept if the successive actuators of usual two-electrode configuration are placed at a very short distance [9].

(C) The authors, published by EDP Sciences. This is an Open Access article distributed under the terms of the Creative Commons Attribution License 4.0 (http://creativecommons.org/licenses/by/4.0/). 
Several innovative designs of multiple DBD-actuators have been proposed to eliminate this damaging cross-talk phenomenon [10]. In design [11], each single two-electrode DBD-actuator in series is replaced by a three-electrode actuator where the third buried electrode is electrically linked with the high-voltage exposed electrode and acts as a shield of one (for example, left) edge of each exposed electrode. Dielectric barrier discharge takes place near opposite (right) edges of the exposed electrodes thereby supporting the volumetric force impact and flow acceleration only in one direction (from left to right) along a streamlined surface. Two dielectric layers separate successively the exposed, the shielding, and the buried grounded electrodes. Note that minimal spatial period of multi-DBDactuators studied experimentally in [11] is $p=20 \mathrm{~mm}$.

Another multi-DBD-actuator consisting of combination of smooth highvoltage buried electrodes, saw-like exposed grounded electrodes, and saw-like exposed floating interelectrodes has been proposed and studied in [12]. Spatial period of the investigated multi-DBD-actuator in [12] is equal to $p=25 \mathrm{~mm}$. More complex design of large-scale multi-DBD-actuator with spatial period of $p=40 \mathrm{~mm}$ has been considered in [13]. Two main features distinguish this design from usual two-electrode one. First, the plane exposed electrodes are replaced by thin wires in order to enhance the volumetric force impact of each single DBD, according to [14]. Second, the potential alteration of the successive both exposed and buried electrodes between high-voltage and ground is used in order to cancel their damaging interference.

The effectiveness of all mentioned designs has been confirmed experimentally. But all of them do not take into account some features of practical application in EGD-LFC. First of all, significant miniaturization of the multiDBD-actuators [11-13] is necessary for their possible application in laminar flow control on a swept wing because of very small boundary layer thickness, as it was mentioned above. Moreover, a precipitation of water on a wing surface in the case of a flight in clouds can lead to uncontrolled gas breakdown in actuators $[12,13]$ where high-voltage swing between adjacent air-exposed electrodes is present. Finally, every multiple DBD-actuator will be installed over a wing skin which must be electricity-conductive according to electrostatic safety requirements. It means that additional dielectric layer separating the wing skin and actuators is necessary in designs [11-13].

\section{PROPOSED DESIGN OF MULTIPLE DIELECTRIC BARRIER DISCHARGE ACTUATOR}

First of all, note that the electric potential acquired by an airplane as a whole in flight due to various electrization processes is determined below conventionally as zero or grounded. The idea of the shielding buried electrodes electrically 


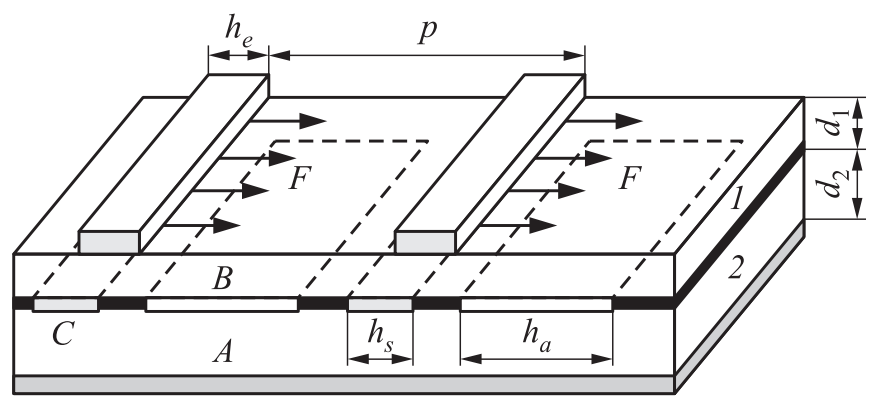

Figure 1 Design of multiple DBD-actuator: $A$ - grounded wing skin; $B$ - grounded accelerating electrodes; $C$ - high-voltage shielding electrodes; $D$ - high-voltage exposed electrodes; and 1 and 2 - dielectric layers

linked with the exposed ones proposed in [11] has been adopted in numerical modeling [15] of slightly modified design of multi-DBD-actuator shown in Fig. 1. Distinctions from design [11] consist, first, in the presence of grounded wing skin $A$ and, second, in the arrangement of the grounded accelerating electrodes $B$ and the high-voltage shielding electrodes $C$ under the same external dielectric layer 1 separating the buried electrodes $B, C$, and the high-voltage exposed electrodes $D$. Electric field strength in the vicinity of the left edges of the exposed electrodes is reduced due to the shielding electrodes and DBD attenuates in these regions. Resulting horizontal volumetric force $F$ generated in DBD near the right edges is directed from left to right. The internal dielectric layer 2 separates electrodes $B, C$, and the wing skin $A$. Numerical modeling in [15] executed for geometric parameters $p=10 \mathrm{~mm}, h_{e}=2 \mathrm{~mm}, h_{s}=2 \mathrm{~mm}, h_{a}=5 \mathrm{~mm}$, $d_{1}=1 \mathrm{~mm}$, and $d_{2}=2 \mathrm{~mm}$ confirmed significant positive influence of the shielding electrodes.

Next evident step in simplification and improvement of multi-DBD design made in [15] consists in the removal of buried accelerating electrodes $B$. In this case, the grounded wing skin acts as common accelerating electrode for all exposed electrodes in series. At the same time, the probability of electric breakdown in glue layer between the electrodes $B$ and $C$ at too small distance between them is removed.

Figure 2 demonstrates the dependencies of the ratios of the maximum values of the electric field strength on the active (right) $E_{1}$ and the passive (left) $E_{2}$ edges of the external electrodes on the width of the shielding electrode at two values of the external dielectric thickness $d_{1}$. The right edge of the shielding electrode is located under the middle of the external one. Further displacement of this edge to the right noticeably weakens the field strength on the right active edge of the external electrode. This influence limits at some extent the possibility of a decrease of the exposed electrode width. The data in Fig. 2 are obtained 


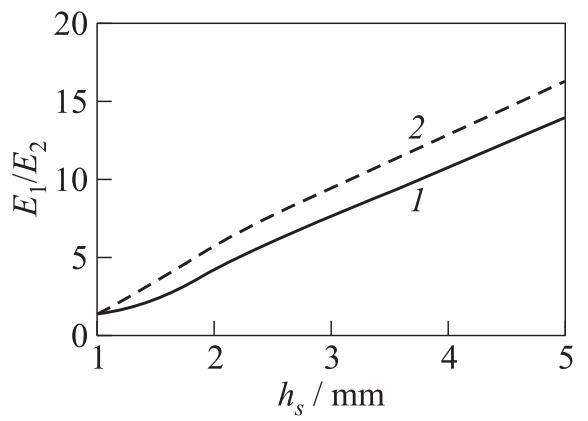

Figure 2 The ratio of the maximums of the electric field strength at the opposite edges of the external electrodes at $d_{1}$ $=0.5(1)$ and $0.25 \mathrm{~mm}(2)$

ternal electrodes at a sufficiently thin external dielectric layer. As a consequence, the more lengthy parts of the grounded electrode $A$ may be involved in generation of a volumetric force than in design with the buried accelerating electrodes $B$ of fixed width.

The operability of the proposed simplified design of multi-DBD-actuator consisting of the common accelerating electrode $A$, the shielding electrodes $C$, and the exposed electrodes $D$ as well as the possibility of its essential miniaturization have been confirmed in experiments described below.

\section{EXPERIMENTAL EQUIPMENT AND METHODS}

The first model of multiple DBD-actuator used in experiments was made of two glass fibre laminate plates, $145 \times 150 \mathrm{~mm}$ in size. The thicknesses of the upper and lower plates were $d_{1}=0.5 \mathrm{~mm}$ and $d_{2}=1 \mathrm{~mm}$, respectively. Nine parallel flat copper electrodes of 50-micron height and 115-millimeter length in span direction were arranged on both sides of the upper plate with spatial period $p=12 \mathrm{~mm}$. The width of the air-exposed electrodes was $h_{e}=2 \mathrm{~mm}$, their height was $50 \mu \mathrm{m}$, and the width of the shielding electrodes was $h_{s}=3 \mathrm{~mm}$. Each shielding electrode was shifted under the nearest exposed one up to $1 \mathrm{~mm}$. The second plate contained a continuous copper electrode on the whole lower surface. The plates were pasted together by special glue under low ambient pressure to avoid possible discharge ignition in air bubbles between the plates.

The airflow velocity induced by the actuator impact in initially quiescent air was measured over the model by Pitot probe made of ceramic pipe with the external and internal diameters of 1.2 and $0.7 \mathrm{~mm}$, respectively. Difference 
between total and static pressures was measured by special inclined alcohol pressure microsensor. When measuring the velocity profiles, the sensor was moved by a traversing microgear at steps of $0.1 \mathrm{~mm}$ normal to the model surface. The model and the traversing microgear were mounted on a common platform. The Pitot probe also could be moved along the center of the model, perpendicular to the external electrodes.

Continuous electrode on the lower plate was grounded and alternating high voltage was applied on air-exposed and shielding electrodes placed on the upper plate. Dielectric barrier discharge on the model was excited by high-voltage power supply generating alternating pulses with the amplitude $V_{h}=1.5-6 \mathrm{kV}$ and the duration $t_{\mathrm{imp}}=20 \mu \mathrm{s}$. The repetition rate of pulses, i. e., the discharge frequency, was controlled by variation of the pause duration $t_{\text {int }}$ between the pulses and could be varied in the range $f \equiv 0.5 /\left(t_{\text {imp }}+t_{\text {int }}\right)=2.5-12.5 \mathrm{kHz}$.

The power supply consisted of two main units - the power module and the module of high-voltage pulses generation. The power module consisting of a supply-line filter and thyristor rectifier was, in essence, the controllable directcurrent source with output controlling voltage $V_{c}=20-250 \mathrm{~V}$. Controlling direct current $I_{c}$ and voltage $V_{c}$ were measured by proper devices arranged in the power supply module.

The module of generation of high-voltage pulses consisted of two units the module of excitation and the module of pulses formation. The excitation module was intended for generation of two series of pulses and control of their parameters $t_{\text {imp }}$ and $t_{\text {int }}$. A quartz oscillator with frequency $1 \mathrm{MHz}$ was used as a source of initial pulses ensuring high stability of the prescribed parameters. The formation of proper high-voltage pulses supplied on the model of DBD-actuator was executed in the bridge transformer circuit containing power high-voltage

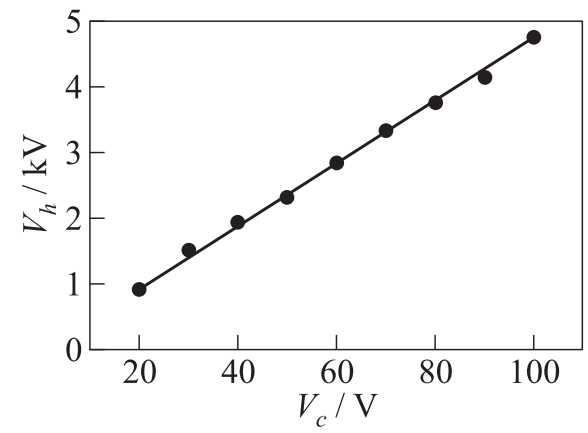

(a)

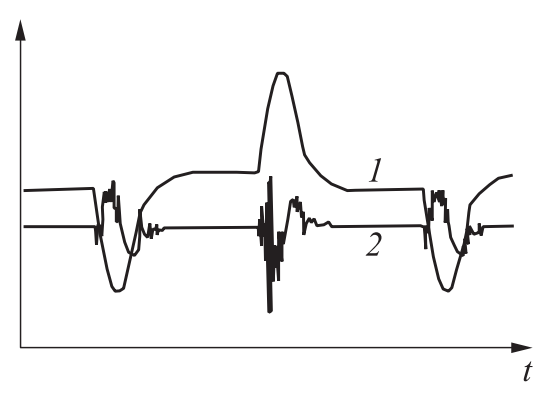

(b)

Figure 3 Dependence of high-voltage amplitude on controlling voltage $(a)$ and typical oscillograms of high voltage (1) and current (2) (b) 
fieldistors. Control of forms and values of the high voltage $V_{h}$ and current $I_{h}$ in the secondary circuit of the transformer was realized by digital oscilloscope of series RIGOL DS1062C.

Figure $3 a$ demonstrates that the amplitude of the high voltage $V_{h}$ applied to the model depends linear on the controlling voltage $V_{c}$ supplied by the directcurrent source. The slope angle of the approximating line does not depend on the frequency $f$ because this angle is determined by relation of turns in transformer coils. In the present case, $V_{h} / V_{c}=47$. As an example, the oscillograms of high voltage $V_{h}$ and total (displacement plus conduction) current $I_{h}$ in the secondary circuit of the transformer are shown in Fig. $3 b$ for $t_{\text {imp }}=20 \mu \mathrm{s}$ and $t_{\text {int }}=60 \mu \mathrm{s}$, i. e., $f=6.25 \mathrm{kHz}$.

\section{MEASUREMENTS OF THE INDUCED FLOW VELOCITY}

The component of the flow velocity induced by multi-DBD-actuator in initially quiescent air directed along the model surface was measured by pressure probe placed on the surface and moving along it perpendicular to the exposed electrodes, i. e., approximately at the height $y=0.6 \mathrm{~mm}$. The results of measurements at three values of the discharge frequency $f=4.16,5$, and $6.25 \mathrm{kHz}$ are shown in Fig. 4. The amplitude of high-voltage pulses was equal to $V_{h}=4.2 \mathrm{kV}$ at the controlling voltage $V_{c}=90 \mathrm{~V}$. Note that the current value in low-voltage circuit of power supply is proportional to the discharge frequency and is determined in this case by the relation $I_{c} / f \approx 0.26 \mathrm{~A} / \mathrm{kHz}$.

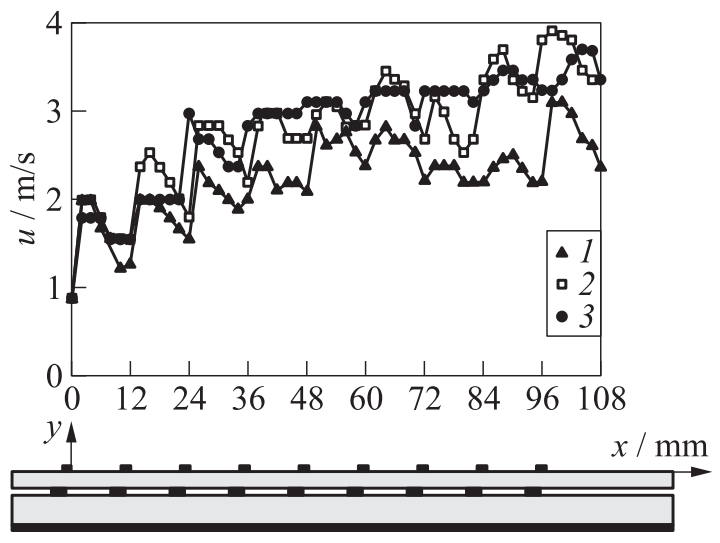

Figure 4 Induced flow velocity along the model surface at $y=0.6 \mathrm{~mm}$ and frequency $f=4.16(1), 5(2)$, and $6.25 \mathrm{kHz}(3)$ 
First of all, note that the discharge glow was observed visually only on the right unshielded edges of all exposed electrodes. The dependences of the induced velocity on the streamwise coordinate $x$ presented in Fig. 4 are nonmonotonic but this velocity always remains positive similar to measurements in [11-13]. It means that the force impact of the actuator is directed in one side (from left to right) over the whole model.

According to Fig. 4, it seems that induced flow achieves the saturation state after the third-fifth single actuator depending on the discharge frequency. To clarify this case, Fig. 5 presents the velocity distributions in the vertical direction measured after the first, second, third, and ninth exposed electrodes at $4 \mathrm{~mm}$ from their right edges (i. e., at $x=4,16,28$, and $100 \mathrm{~mm}$ ) at high-voltage amplitude $V_{h}=4.2 \mathrm{kV}$ and frequency $f=6.25 \mathrm{kHz}$. Indeed, the maximal induced velocity increases fast from $1.5 \mathrm{~m} / \mathrm{s}$ after the first actuator up to $2.4 \mathrm{~m} / \mathrm{s}$ after the third one and only to $3 \mathrm{~m} / \mathrm{s}$ after the ninth actuator.

But the maximum of the induced velocity is not the essential characteristic of plasma actuators because it depends, for example, on external overflow. The intrinsic characteristics of plasma actuators determinative their effectiveness for flow control are the time averaged and spatial integrated volumetric force created by actuator and the average consumed electric power. The velocity distributions presented in Fig. 5 permit to estimate the algebraic sum of the positive volumetric force generated by actuator and the negative skin friction force incipient due to flow appearance.

Simplest integral momentum method applicable for pressure probe measurements [14] was used for total force calculation. The results are presented in Fig. 6. One can see that the sum of volumetric and skin friction forces increases almost linear after the first three actuators but then its growth becomes much slower. It seems evident that the volumetric force generated by actu-

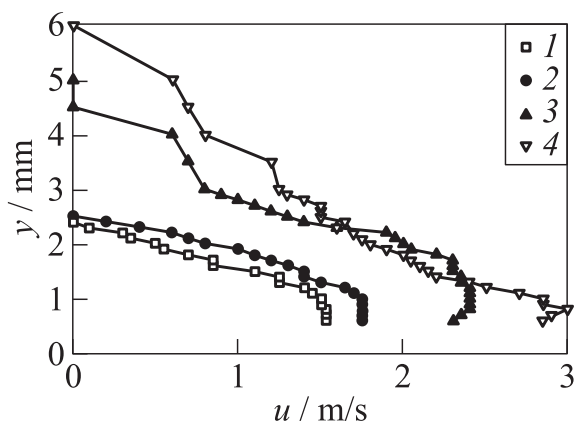

Figure 5 Distributions of the induced flow velocity after the first (1), second (2), third (3), and ninth (4) single actuators

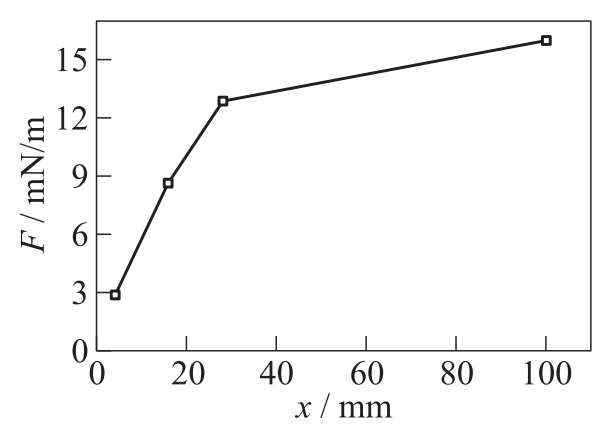

Figure 6 Distribution of total induced force along the model 
ators must increase along the model proportionally to the actuator number. But the integral of the friction force along the model surface increases modulo more rapidly than linear because of gradual increase in the vertical gradient of flow velocity on the model surface and, hence, local skin friction. A very significant contribution of viscous drag in total thrust produced by single DBDactuator was emphasized in [16]. Local skin friction can be measured by more complex and precise methods than used here, for example, by particle image velocimetry [17]. Nevertheless, a crude estimate can be obtained using the data of Fig. 5. The average skin friction between the third and ninth electrodes is estimated as $\tau_{w} \approx \mu \Delta u / \Delta y$ where $\mu \approx 1.8 \cdot 10^{-5} \mathrm{~kg} /(\mathrm{m} \cdot \mathrm{s}), \Delta u \approx 2 \mathrm{~m} / \mathrm{s}$, and $\Delta y \approx 5 \cdot 10^{-4} \mathrm{~m}$; hence, $\tau_{w} \approx 0.07 \mathrm{~N} / \mathrm{m}^{2}$. Local skin friction is integrated along the part of the surface $\Delta x \approx 0.07 \mathrm{~m}$; hence, the integral friction force is estimated as $F_{f}=-\tau_{w} \Delta x \approx-5 \cdot 10^{-3} \mathrm{~N} / \mathrm{m}$ and is modulo comparable with the values of the total force in Fig. 5, i. e., really contributes noticeably in this force.

\section{SIMPLE METHOD OF THE CONSUMED ELECTRIC POWER MEASUREMENT}

Two methods are usually used for computation of the electric power consumed by DBD-actuator [10]. The first one consists in time-averaged integration of the product of current and voltage applied to actuator over several cycles of the discharge. The main problem of this method is related with the presence of high current peaks; therefore, high enough resolution in current measurements is necessary. Another method is based on placing a capacitor between grounded electrode and earth, plotting the voltage-charge curve (usually called "Lissajous loop") and calculation of the area of this curve corresponding to the energy dissipated by the discharge during one cycle.

The features of the high-voltage power source used in the current experiments permit to estimate the electric power consumed by the discharge simply and with acceptable accuracy immediately during an experiment at variation of its parameters, for example, amplitude and frequency of the applied voltage, ambient pressure, etc. Controlling voltage $V_{c}$ and current $I_{c}$ at the output of the rectifier module gives the value of the total electric power $P_{\text {tot }}=I_{c} V_{c}$ consumed, at first, by the module of generation of high-voltage pulses and, at second, by the discharge on the model of multi-DBD-actuator, i. e., $P_{\text {tot }}=P_{\text {gen }}+P_{\text {dis }}$. If the model is cut off from power supply, the total power is consumed only by the module of pulses generation, i. e., $P_{\text {tot }}=P_{\text {gen }}$. Preliminary measurements of $P_{\text {gen }}$ with the model in the silent state at various controlling voltage $V_{c}$ permit henceforth to calculate the discharge power as $P_{\text {dis }}=P_{\text {tot }}-P_{\text {gen }}$.

Figure 7 represents the dependences of the consumed power on controlling voltage at the discharge frequency $f=6.25 \mathrm{kHz}$ corresponding to the results 

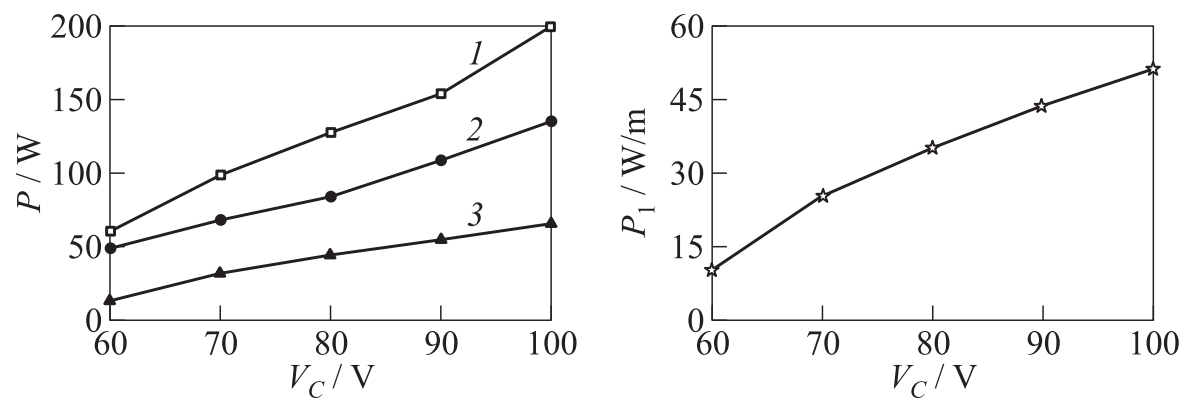

Figure 7 Dependences of the consumed electric power on controlling voltage at $f=6.25 \mathrm{kHz}: 1-P_{\text {tot }} ; 2-P_{\text {gen }} ;$ and $3-P_{\text {dis }}$

shown in Figs. 5 and 6 . Here, the power consumed by the discharge per unit length of the exposed electrodes $P_{1}$ is shown too. In particular, $P_{1}=44 \mathrm{~W} / \mathrm{m}$ at the controlling voltage $V_{c}=90 \mathrm{~V}$ corresponding to the high-voltage amplitude $V_{h}=4.2 \mathrm{kV}$.

The data of Figs. 6 and 7 permit to estimate the average energy efficiency $E$ of the considered multi-DBD-actuator defined as the relation of average total force to consumed power per unit length of the exposed electrodes [18]. The unit average force is evaluated here as one third of the force measured behind the third electrode $F_{1}=12.9 / 3=4.3 \mathrm{mN} / \mathrm{m}$. As $P_{1}=44 \mathrm{~W} / \mathrm{m}$ at $V_{h}=4.2 \mathrm{kV}$ and $f=6.25 \mathrm{kHz}$, then $E \approx 10^{-4} \mathrm{~s} / \mathrm{m}$. This value is appreciably less than the best effectiveness $\left(E \approx 6 \cdot 10^{-4} \mathrm{~s} / \mathrm{m}\right)$ achieved in single DBD-actuator [10]. Moreover, the average force density per unit of a streamlined surface obtained in the considered actuator is estimated as $\langle F\rangle \equiv F_{1} / p=4.3 / 12=0.36 \mathrm{~N} / \mathrm{m}^{2}$. According to the numerical estimations [19], the EGD-LFC method demands the value of the average volumetric force density about $\langle F\rangle=(3-4) \mathrm{N} / \mathrm{m}^{2}$. It means that further improvement and optimization of the considered multi-DBDactuator is necessary.

\section{LIMITATIONS OF MULTIPLE DIELECTRIC BARRIER DISCHARGE ACTUATOR MINIATURIZATION}

The possibility of significant miniaturization of the proposed design of multiDBD-actuator was verified. Figure $8 a$ shows the model with two multiple actuators made of the same materials as above, i. e., the upper and lower dielectric plates thicknesses are $d_{1}=0.5 \mathrm{~mm}$ and $d_{2}=1 \mathrm{~mm}$. The width of both external and shielding electrodes is $h_{e}=h_{s}=1 \mathrm{~mm}$. The shielding electrodes 


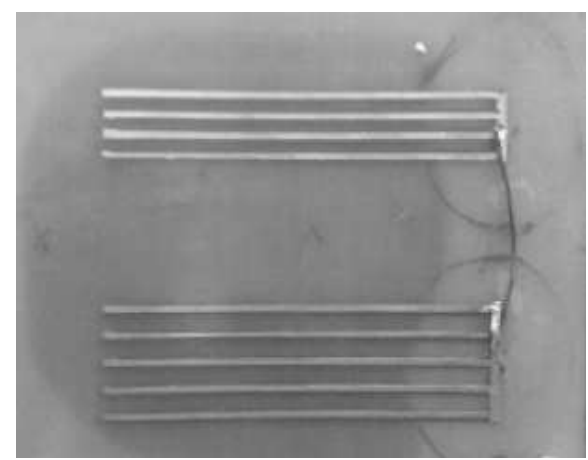

(a)
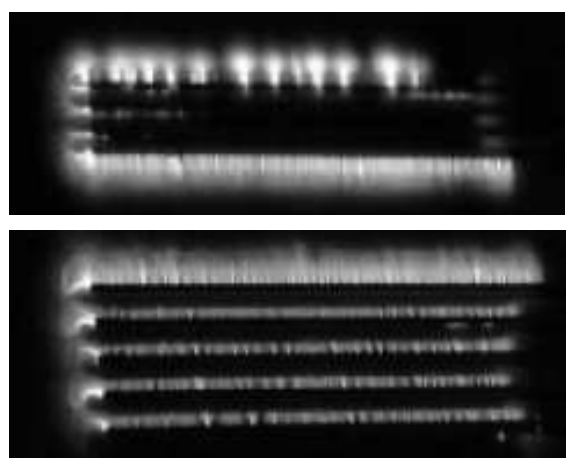

(b)

Figure 8 Photos of multi-DBD-actuators $(a)$ and the discharge $(b)$ at period $p$ $=4 \mathrm{~mm}$ (top) and $5 \mathrm{~mm}$ (below)

in Fig. $8 a$ are placed under the lower edges of the exposed electrodes and are shifted upward at the middle of the latest. That is the upper edges of the exposed electrodes must be active. The difference between the actuators consists in the spatial period: $p=4 \mathrm{~mm}$ in the upper actuator and $p=5 \mathrm{~mm}$ in the lower one.

The photos in Fig. $8 b$ demonstrate that the active edges of the lower actuator $(p=5 \mathrm{~mm})$ act by desired manner creating the force impact bottom-up. The picture of the discharge in the upper actuator $(p=4 \mathrm{~mm})$ at the same applied

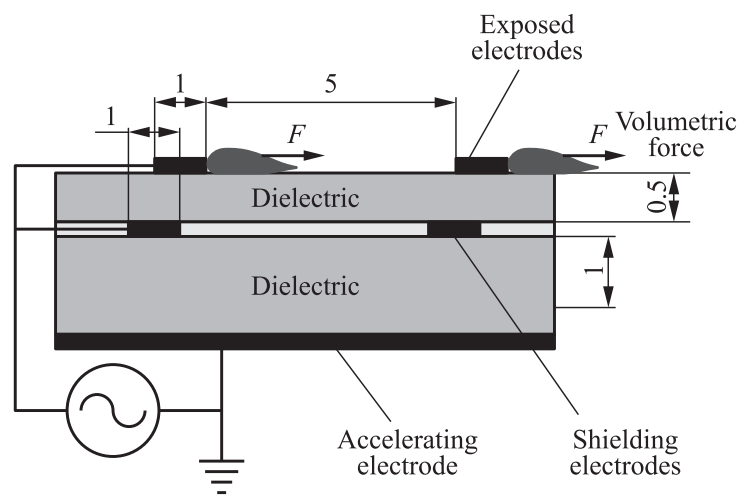

(a)

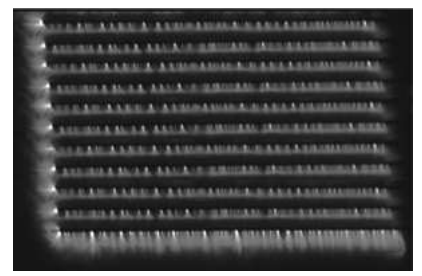

(b)

Figure 9 Scheme of multi-DBD-actuator $(a)$ and photo of the discharge on 11 exposed electrodes at $p=6 \mathrm{~mm}(b)$. Dimensions are in millimeters 
voltage is quite different. In this case, the discharge takes place even on the shielded edge of the lowermost electrode and is almost hidden on active edges of the first three lower electrodes. One can hope that the operable multiple actuator with the period less than $5 \mathrm{~mm}$ can be made by a decrease of the thicknesses of the dielectric layers.

Accounting for previous results, the model of multi-DBD-actuator containing 12 couples of the exposed and shielding electrodes with sizes indicated in Fig. $9(p=6 \mathrm{~mm})$ has been made. The photo in Fig. $9 b$ shows the discharge picture at the applied voltage of $8 \mathrm{kV}$ and $10 \mathrm{kHz}$. It is evident that the discharge takes place only on all active (lower) edges of the exposed electrodes and generates the force impact and the induced flow top-down.

\section{CONCLUDING REMARKS}

The preliminary experimental study revealed the potentiality of the proposed design of multi-DBD-actuator for application in electrogasdynamic laminar flow control on a swept wing.

\section{REFERENCES}

1. Moreau, E. 2007. Airflow control by non-thermal plasma actuators. J. Phys. D Appl. Phys. 40:605-636.

2. Corke, T. C., M. L. Post, and D. M. Orlov. 2007. SDBD plasma enhanced aerodynamics: Concepts, optimization and applications. Prog. Aerosp. Sci. 43:193-217.

3. Corke, T. C., C. L. Enloe, and S.P. Wilkinson. 2010. Dielectric barrier discharge plasma actuators for flow control. Annu. Rev. Fluid Mech. 42:505-529.

4. Wang, J. J., K-S. Choi, L-H. Feng, T. N. Jukes, and R. D. Whalley. 2013. Recent developments in DBD plasma flow control. Prog. Aerosp. Sci. 62:52-78.

5. Chernyshev, S. L., A.Ph. Kiselev, and A.P. Kuryachii. 2011. Laminar flow control research at TsAGI: Past and present. Prog. Aerosp. Sci. 47:169-185.

6. Kuryachii, A.P., and S. V. Manuilovich. 2011. Attenuation of cross-flow-type instability in a $3 \mathrm{D}$ boundary layer due to volumetric force impact. TsAGI Sci. J. 42:345-360.

7. Roth, J. R., and D. M. Sherman. 1998. Boundary layer flow control with a one atmosphere uniform glow discharge surface plasma. AIAA Paper No. 98-328.

8. Chernyshev, S. L., A.P. Kuryachii, S. V. Manuilovich, D. A. Rusyanov, and V.V. Skvortsov. 2013. Attenuation of cross-flow-type instability in compressible boundary layer by means of plasma actuators. AIAA Paper No. 2013-321.

9. Do, H., W. Kim, M. A. Capelli, and M. G. Mungal. 2008. Cross-talk in multiple dielectric barrier discharge actuators. Appl. Phys. Lett. 92:071504. 
10. Benard, N., and E. Moreau. 2014. Electrical and mechanical characteristics of surface AC dielectric barrier discharge plasma actuators applied to airflow control. Exp. Fluids 55:1846.

11. Benard, N., A. Mizuno, and E. Moreau. 2009. A large-scale multiple dielectric barrier discharge actuator based on an innovative three-electrode design. J. Phys. D Appl. Phys. 42:235204.

12. Berendt, A., J. Podlin'ski, and J. Mizeraczyk. 2011. Elongated DBD with floating interelectrodes for actuators. Eur. Phys. J. Appl. Phys. 55:13804.

13. Debien, A., N. Benard, and E. Moreau. 2012. Streamer inhibition for improving force and electric wind produced by DBD actuators. J. Phys. D Appl. Phys. 45:215201.

14. Hoskinson, A., N. Hershkowitz, and D. Ashpis. 2009. Comparisons of force measurement methods for DBD plasma actuators in quiescent air. AIAA Paper No. 2009485.

15. Kuryachii, A.P., D. A. Rusyanov, S. L. Chernyshev, and V. V. Skvortsov. 2013. About increase of efficiency of plasma multi-actuator system for boundary layer control. TsAGI Sci. J. 44:305-326.

16. Font, G. I., C. L. Enloe, and T. E. McLaughlin. 2009. Effect of volumetric momentum addition on the total force production by a plasma actuator. AIAA Paper No. 2009-4285.

17. Kriegseis, J., C. Schwarz, A. Duchmann, S. Grundmann, and C. Tropea. 2012. PIVbased estimation of DBD plasma-actuator force terms. AIAA Paper No. 2012-411.

18. Porter, C. O., J. W. Baughn, T. E. McLaughlin, C. L. Enloe, and G. I. Font. 2006. Temporal force measurements on an aerodynamic plasma actuator. AIAA Paper No. 2006-104.

19. Chernyshev, S. L., A.P. Kuryachii, S. V. Manuilovich, D. A. Rusyanov, and M. D. Gamirullin. 2014. Theoretical modeling of the electrogasdynamic method of laminar flow control on a swept wing. ICAS 2014 Proceedings. Paper 2014_0303. 